\title{
The value of hemoglobin-to-red blood cell distribution width ratio (Hb/RDW), neutrophil-to- lymphocyte ratio (NLR), and platelet-to-lymphocyte ratio (PLR) for the diagnosis of nasopharyngeal cancer
}

\section{Zhongyuan Lin Lin}

People's Hospital of Guangxi Zhuang Autonomous Region

\section{Xuan Zhang}

Guangxi Medical University First Affiliated Hospital

\section{Songshan Zhu}

People's Hospital of Guangxi Zhuang Autonomous Region

Yu Luo

People's Hospital of Guangxi Zhuang Autonomous Region

\section{Yanyun Chen}

People's Hospital of Guangxi Zhuang Autonomous Region

Xiaochun Liu ( $\sim 2548730251 @ q q . c o m$ )

People's Hospital of Guangxi Zhuang Autonomous Region

\section{Research article}

Keywords: hemoglobin-to-red blood cell distribution width, neutrophil-to-lymphocyte ratio, platelet-tolymphocyte ratio, nasopharyngeal cancer

Posted Date: January 28th, 2020

DOI: https://doi.org/10.21203/rs.2.22048/v1

License: (c) (1) This work is licensed under a Creative Commons Attribution 4.0 International License. Read Full License

Version of Record: A version of this preprint was published at Medicine on July 16th, 2021. See the published version at https://doi.org/10.1097/MD.0000000000026537. 


\section{Abstract}

Background: The clinical significance of hemoglobin-to-red blood cell distribution width (Hb/RDW) for the diagnosis of nasopharyngeal cancer (NPC) has not been reported yet. This study aimed to evaluate the value of preoperative $\mathrm{Hb} / \mathrm{RDW}$, neutrophil-to-lymphocyte ratio (NLR), and platelet-to-lymphocyte ratio (PLR) for the diagnosis of NPC.

Methods: A total of 180 NPC patients (NPC group) and 149 healthy subjects (control group) were recruited to assess the value of Hb/RDW, NLR, and PLR for the diagnosis of NPC.

Results: It was noted that NLR and PLR were significantly higher in the NPC group than those in the control group $(P<0.001)$, however, $\mathrm{Hb} / \mathrm{RDW}$ was lower in the NPC group compared with that in the control

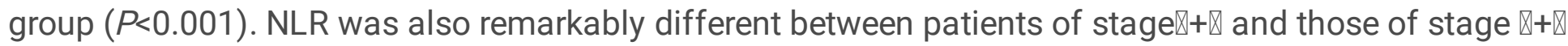
$(P=0.043)$, and that was different in patients with lymph node metastases or not $(P=0.030)$. Besides, $P L R$ was significantly different in patients with serosal invasion or not $(P=0.031)$.Compared with $\mathrm{Hb} / \mathrm{RDW}$ alone (sensitivity, $66.67 \%$; specificity, $85.23 \%)$, the sensitivity $(67.78 \%, 72.78 \%)$ and specificity $(89.62 \%$, $90.6 \%)$ of $\mathrm{Hb} / \mathrm{RDW}$ with NLR and PLR were both increased. Furthermore, $\mathrm{Hb} / \mathrm{RDW}$ combined with NLR (area under the receiver operating characteristic curve (ROC) (AUC), 0.824; 95\% confidence interval (Cl):0.779-0.864, $P=0.0080$ ) or PLR (AUC:0.851, 95\%Cl:0.808-0.888, $P=0.0002$ ) had a greater AUC value for the diagnosis of NPC compared with Hb/RDW alone (AUC: $0.781,95 \% \mathrm{Cl}: 0.732-0.824$ ).

Conclusion: $\mathrm{Hb} / \mathrm{RDW}$ can be used as a valuable indicator for the diagnosis of NPC. Preoperative $\mathrm{Hb} / \mathrm{RDW}$ combined with NLR or PLR had higher value for the diagnosis of NPC.

\section{Background}

Nasopharyngeal cancer (NPC) is a common malignant tumor of the nasopharynx in southern China and Southeast Asia. Its incidence ranks the first in head and neck cancer, especially in Guangdong, with the incidence of $20-30$ patients per 100,000 people ${ }^{[1-4]}$. NPC is highly metastatic and invasive, while it is sensitive to both chemotherapy and radiotherapy. In China, about 60,600 new cases are annually diagnosed as NPC, of which 34,100 patients with NPC pass away ${ }^{[5]}$. Due to the clinical heterogeneity of NPC, early diagnosis of NPC is a main challenge in the majority of patients. Although new methods for early diagnosis of NPC have been presented, the majority of cases are diagnosed at late stage. Symptoms of NPC are gradually worsen, and about one-fifth of the patients may develop local recurrence or distant metastasis ${ }^{[6]}$. Therefore, searching for more credible early indicators has become a hot spot in clinical diagnosis of NPC. In recent years, a significant progress has been made in the study of hematological markers for prognosis of different tumors. The hematological indices have been widely used as indicators for prognosis, which are highly reproducible and cost-effective for clinical use. This study aimed to investigate the clinical significance of hemoglobin-to-red cell distribution width ratio (Hb/RDW), neutrophil-to-lymphocyte ratio (NLR), and platelet-to-lymphocyte ratio (PLR) in prevention and 
diagnosis of NPC. It is noteworthy that we, for the first time used the above-mentioned indicators for the diagnosis of NPC.

\section{Patients And Methods}

\section{Patients}

The medical records of 180 patients (165 (91.67\%) were males; mean age, $54.53 \pm 11.05$ years old), who were pathologically diagnosed as NPC in our hospital between December 2010 and September 2019, were retrospectively reviewed. Exclusion criteria: (a) previous treatment; (b) other malignant tumors; (c) multiple infections; (d) blood disorders; (e) anemia;(f) diabetes mellitus; (g) acute and chronic infectious diseases. Additionally, 149 healthy subjects (139 (93.29\%) were male; mean age, 53.65 \pm 10.44 years old) were included in control group. The study protocol was approved by the Ethics Committee of the People's Hospital of Guangxi Zhuang Autonomous Region (Nanning, China).

\section{Methods}

Blood samples were collected in tubes containing ethylenediaminetetraacetic acid (EDTA). Hematology test included measurement of the levels of hemoglobin $(\mathrm{Hb})$, hematocrit $(\mathrm{HCT})$, plateletcrit (PCT), RDW, neutrophil (N), lymphocyte (L), and platelets (PLT) using the Sysmex XN-9000 hematology analyzer (Sysmex Corp., Kobe, Japan) for 30 min after the blood draw was completed. All the patients with NPC were clinically staged in accordance with the 7th edition of the American Joint Committee on Cancer (AJCC) TNM staging manual.

\section{Statistical analysis}

The statistical analysis was undertaken using SPSS19.0software (IBM, Armonk, NY, USA). The normally distributed data were presented as mean \pm standard deviation (SD) or median values, and the categorical data were expressed as percentage. The Tukey's test was employed to evaluate the differences between groups. Pearson's correlation coefficient was used to analyze the correlation between variables in NPC group. In addition, MedCalc 15.0 software was found to be helpful to calculate sensitivity and specificity, and draw receiver operating characteristic (ROC) curves. $P<0.05$ was considered statistically significant.

\section{Results}

\section{Clinical characteristics of patients with NPC and healthy controls}

In short, 180 patients with NPC (165 (91.67\%) males) with a mean age of $54.53 \pm 11.05$ years old, and 149 healthy controls (139 $(93.29 \%)$ males) with a mean age of $53.65 \pm 10.44$ years old were included in this study. There was no marked difference in sex and age between the two groups $(P=0.581, P=0.460$, respectively). 
Results of analysis of differences in laboratory variables between the NPC group and control group are presented in Table 1, and there were significant differences in $\mathrm{Hb}, \mathrm{HCT}, \mathrm{Hb} / \mathrm{RDW}, \mathrm{PCT}, \mathrm{RDW}, \mathrm{NLR}$, and PLR between the two groups $(P<0.05)$. It also was found that NLR and PLR were significantly higher in NPC group than those in control group $(P<0.001$, Fig. 1a, Fig. 1b). However, $\mathrm{Hb} / \mathrm{RDW}$ was noticeably lower in the NPC group compared with that in the control group $(P<0.001$, Fig. $1 \mathrm{c})$.

\section{NLR and PLR in patients with NPC}

The values of NLR and PLR in NPC group are shown in Table 2. It was found that NLR was markedly

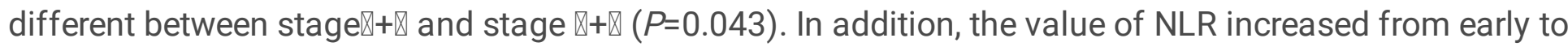
late stages $(P=0.043$, Fig. $2 \mathrm{a})$, and it was significantly different in patients with lymph node metastases or not $(P=0.030$, Fig. 2 b). Moreover, PLR was significantly different in patients with serosal invasion or $\operatorname{not}(P=0.031$, Fig. $2 \mathrm{c})$.

\section{Correlation analysis of NLR, PLR and Hb/RDW in patients with NPC}

Results of correlation analysis of NLR, PLR and Hb/RDW in patients with NPC are presented in Table 3. It was noted that $\mathrm{Hb} / \mathrm{RDW}$ was negatively correlated with NLR and PLR $(P=0.039, \mathrm{r}=-0.154)$; NLR was positively correlated with TNM (tumor, node, metastasis) stage $(P=0.043, \mathrm{r}=-0.151)$, and lymph node metastasis $(P=0.030, \mathrm{r}=-0.162)$, and negatively correlated with distant metastasis $(P=0.002, \mathrm{r}=-0.232)$. Besides, PLR was positively correlated with serosal invasion $(P=0.031, \mathrm{r}=-0.161)$, and negatively correlated with distant metastasis $(P=0.002, \mathrm{r}=-0.227)$.

\section{Diagnostic efficiency of NLR, PLR and Hb/RDW in NPC group and control group}

The diagnostic efficiency of Hb/RDW combined with NLR or PLR in NPC group is shown in Table 4. The sensitivity (66.67\%) of Hb/RDW and the specificity (89.28\%) of PLR for the diagnosis of NPC were both the highest. Compared with Hb/RDW alone (sensitivity: $66.67 \%$; specificity: $85.23 \%$ ), the sensitivity $(67.78 \%, 72.78 \%)$ and specificity $(89.62 \%, 90.6 \%)$ of $\mathrm{Hb} / \mathrm{RDW}$ combined with NLR and PLR, which were used for the diagnosis of NPC, respectively, were separately increased. Furthermore, $\mathrm{Hb} / \mathrm{RDW}$ combined with NLR (area under the curve (AUC):0.824, 95\% confidence interval $(\mathrm{Cl}): 0.779-0.864, P=0.0080$ ) or PLR (AUC:0.851, 95\% Cl:0.808-0.888, $P=0.0002$ ) had a greater AUC value for the diagnosis of NPC compared with $\mathrm{Hb} / \mathrm{RDW}$ alone (AUC: $0.781,95 \% \mathrm{Cl}$ : 0.732-0.824, Fig. 3).

\section{Discussion}

NPC is a common cancer in southern China, seriously endangering human health. Hence, exploration of further reliable early diagnostic indicators is highly significant to improve the diagnosis of NPC. Recent studies demonstrated that chronic inflammation is one of the biological characteristics of tumors, and inflammation is associated with the development of NPC ${ }^{[7-9]}$. NLR and PLR were previously found as 
indicators of inflammation for the diagnosis or prognosis of NPC ${ }^{[10]}$. Liao et al. ${ }^{[11]}$ studied the influence of NLR elevation on the prognosis of patients with NPC. Yang et al. ${ }^{[12]}$ found that high levels of PLR were associated with poor prognosis of NPC. The mechanism of NPC influenced by NLR and PLR may be that systemic inflammatory responses stimulate tumor metastasis via promoting angiogenesis and inhibiting apoptosis. Lymphocytes are immune cells found in the blood and lymph tissues, and T and B lymphocytes are the two main types. It is well-known that tumor-infiltrating lymphocytes (TILs) in tumors can attack and eradicate tumor cells in the cancer patients. Inflammatory chemokines are mainly produced in non-lymphoid organs and primarily affect cells of the innate immune system, while they may influence different types of effector and memory lymphocytes ${ }^{[13-14]}$. Platelets, as a key source of circulating angiogenesis-related proteins, can regulate tumor cell growth and angiogenesis, thereby accelerating reproduction and metastasis of tumor cells ${ }^{[15,16]}$.

In the present study, the values of NLR and PLR in the NPC group were significantly higher than those in the control group. NLR was found to be negatively correlated with distant metastasis $(P=0.002$, $r=-0.232)$, while it was positively correlated with lymph node metastasis $(P=0.030, r=-0.162)$, and TNM stage $(P=0.043, r=-0.151)$, which appeared as an appropriate marker of disease severity, as its value was markedly elevated with worsening of NPC. Additionally, PLR was negatively correlated with distant metastasis $(P=0.002, r=-0.227)$, whereas positively correlated with serosal invasion $(P=0.031, r=-0.161)$. The above-mentioned results indicated that NLR and PLR are closely associated with the clinicopathological characteristics of NPC patients, demonstrating that these two indicators can guide the clinical pathological staging of NPC.

To our knowledge, anemia is a common complication of myelosuppressive chemotherapy that results in a decreased functional capacity and quality of life for cancer patients. Anemia can be caused by blood loss, decreased red blood cell production, and increased red blood cell breakdown. Hemoglobin $(\mathrm{Hb})$ is taken as a diagnostic indicator of anemia, and is used for the prognostic evaluation of lung cancer patients ${ }^{[17]}$. However, RDW is an indicator of the type of anemia, which is associated with the aggressiveness of advanced tumors ${ }^{[10,18,19]}$, and is used for differential diagnosis of tumors as well[20] High values of RDW were correlated with advanced tumor stage and invasiveness in patients with nonsmall cell lung cancer (NSCLC) ${ }^{[10]}$ and kidney cancer ${ }^{[19]}$. It has been reported that low levels of Hb may accelerate tumor angiogenesis by causing hypoxia ${ }^{[21-23]}$. Additionally, a low $\mathrm{Hb}$ level is a known prognostic marker for patients with oral squamous cell carcinoma ${ }^{[24]}$. In recent years, a number of scholars demonstrated that $\mathrm{Hb} / \mathrm{RDW}$ is associated with survival of patients with advanced NSCLC ${ }^{[10]}$, and can be used as a marker for prognosis of esophageal cancer as well ${ }^{[26]}$. A number of scholars reported that $\mathrm{Hb} / \mathrm{RDW}$ presents further reliable information compared separately with $\mathrm{Hb}$ and RDW, eliminating potential bias. The results of the present study showed that although there was no correlation between $\mathrm{Hb} / \mathrm{RDW}$ and TNM stage of NPC, the mean Hb/RDW in the NPC group was significantly lower than that in the control group. Moreover, $\mathrm{Hb} / \mathrm{RDW}$ was found to be negatively correlated with NLR and $\operatorname{PLR}(P=0.039, r=-0.154)$. 
According to the results of ROC curve analysis, $\mathrm{Hb} / \mathrm{RDW}$ had the highest sensitivity for the diagnosis of NPC, and PLR had the highest specificity for the diagnosis of NPC. Compared with Hb/RDW alone, sensitivity and specificity were increased when Hb/RDW was combined with NLR or PLR for the diagnosis of NPC, respectively. In addition, $\mathrm{Hb} / \mathrm{RDW}$ combined with NLR or PLR led to a greater AUC for the diagnosis of NPC compared with $\mathrm{Hb} / \mathrm{RDW}$ alone, demonstrating that $\mathrm{Hb} / \mathrm{RDW}$ in combination with NLR or NLR can be used for the diagnosis of NPC. A combined detection approach improves the specificity and sensitivity, thereby elevating the diagnostic value of NPC. However, further large-scale prospective studies need to be conducted to elucidate the underlying mechanism.

In summary, $\mathrm{Hb} / \mathrm{RDW}$ combined with NLR or PLR maybe associated with a higher sensitivity and specificity for early diagnosis of NPC. However, there are a number of deficiencies that need to be elucidated by further conducting large sample studies with longer duration of follow-up. This is the first time that $\mathrm{Hb} / \mathrm{RDW}$ is used for the diagnosis of NPC, and NLR and PLR combined with Hb/RDW have higher efficiency for the diagnosis of NPC.

\section{Conclusions}

$\mathrm{Hb} / \mathrm{RDW}$ can be used as a valuable indicator for the diagnosis of NPC patients. Preoperative Hb/RDW combined with NLR or PLR had higher value for the diagnosis of NPC.

\section{Declarations}

\section{- Ethics approval and consent to participate}

The study was approved by the Ethics Committee of The People's Hospital of Guangxi Zhuang Autonomous Region. All the participants gave written informed consent.

\section{- Consent for publication}

Not applicable.

\section{- Availability of data and materials}

The datasets used and/or analysed during the current study are available from the corresponding author on reasonable request.

\section{- Competing interests}

The authors declare that they have no competing interests.

\section{- Funding}

This research received no external funding. 


\section{- Authors' contributions}

LZY. and ZX. collated study data, assisted with the study data analysis, and wrote the manuscript. ZSS. and LY. conceived the idea and designed the study. CYY carried out the statistical analysis and interpreted the results. LXC. revised the manuscript. All authors reviewed the manuscript and agreed to submit.

\section{- Acknowledgements}

We highly appreciate the efforts dedicated by staffs at Department of Clinical Laboratory, the People's Hospital of Guangxi Zhuang Autonomous Region, Nanning, China.

\section{Abbreviations}

AUC, area under the receiver operating characteristic (ROC) curve; $95 \% \mathrm{Cl}, 95 \%$ confidence interval; $\mathrm{Hb} / \mathrm{RDW}$, hemoglobin/red cell distribution width (Hb/RDW); PCT, plateletcrit; PLR, platelet-to-lymphocyte ratio; NLR, neutrophil-to-lymphocyte; NPC, nasopharyngeal cancer.

\section{References}

1. Su Z, Mao YP, OuYang PY, et al. Initial Hyperleukocytosis and Neutrophilia in Nasopharyngeal Carcinoma: Incidence and Prognostic Impact[J]. PLoS One. 2015;10:e0136752.

2. He JR, Shen GP, Ren ZF, et al. Pretreatment levels of peripheral neutrophils and lymphocytes as independent prognostic factors in patients with nasopharyngeal carcinoma[J]. Head Neck. 2012;34:1769-1776.

3. Parkin DM, Bray F, Ferlay J, et al. Global cancer statistics, 2002[J]. CA Cancer J Clin. 2005;55:74-108.

4. Schreiber RD, Old LJ,Smyth MJ. Cancer immunoediting: integrating immunity's roles in cancer suppression and promotion[J]. Science. 2011;331:1565-1570.

5. Chen W, Zheng R, Baade PD, et al. Cancer statistics in China, 2015[J]. CA Cancer J Clin. 2016;66:115132.

6. Lee AW, Lau WH, Tung SY, et al. Preliminary results of a randomized study on therapeutic gain by concurrent chemotherapy for regionally-advanced nasopharyngeal carcinoma: NPC-9901 Trial by the Hong Kong Nasopharyngeal Cancer Study Group[J]. J Clin Oncol. 2005;23:6966-6975.

7. Liao Q, Zeng Z, Guo X, et al. LPLUNC1 suppresses IL-6-induced nasopharyngeal carcinoma cell proliferation via inhibiting the Stat3 activation[J]. Oncogene. 2014;33:2098-2109.

8. Hsu MC, Hsiao JR, Chang KC, et al. Increase of programmed death-1-expressing intratumoral CD8 T cells predicts a poor prognosis for nasopharyngeal carcinoma[J]. Mod Pathol. 2010;23:1393-1403.

9. Yip WK, Abdullah MA, Yusoff SM, et al. Increase in tumour-infiltrating lymphocytes with regulatory $T$ cell immunophenotypes and reduced zeta-chain expression in nasopharyngeal carcinoma patients[J]. Clin Exp Immunol. 2009;155:412-422. 
10. Warwick R, Mediratta N, Shackcloth M, et al. Preoperative red cell distribution width in patients undergoing pulmonary resections for non-small-cell lung cancer[J]. Eur J Cardiothorac Surg. 2014;45:108-113.

11. Liao LJ, Hsu WL, Wang CT, et al. Prognostic impact of pre-treatment neutrophil-to-lymphocyte ratio (NLR) in nasopharyngeal carcinoma: A retrospective study of 180 Taiwanese patients[J]. Clin Otolaryngol. 2018;43:463-469.

12. Yang S, Zhao K, Ding X, et al. Prognostic Significance of Hematological Markers for Patients with Nasopharyngeal Carcinoma: A Meta-analysis[J]. J Cancer. 2019;10:2568-2577.

13. Fridlender ZG, Sun J, Kim S, et al. Polarization of tumor-associated neutrophil phenotype by TGFbeta: "N1" versus "N2" TAN[J]. Cancer Cell. 2009;16:183-194.

14. Grivennikov SI, Greten FR,Karin M. Immunity, inflammation, and cancer[J]. Cell.2010;140:883-899.

15. Sharma D, Brummel-Ziedins KE, Bouchard BA, et al. Platelets in tumor progression: a host factor that offers multiple potential targets in the treatment of cancer[J]. J Cell Physiol. 2014;229:1005-1015.

16. Okamoto E, Osaki M, Kase S, et al. Thymidine phosphorylase expression causes both the increase of intratumoral microvessels and decrease of apoptosis in human esophageal carcinomas[J]. Pathol Int. 2001;51:158-164.

17. Aoe K, Hiraki A, Maeda T, et al. Serum hemoglobin level determined at the first presentation is a poor prognostic indicator in patients with lung cancer[J]. Intern Med. 2005;44:800-804.

18. Seretis $C$, Seretis F, Lagoudianakis $E$, et al. Is red cell distribution width a novel biomarker of breast cancer activity? Data from a pilot study[J]. J Clin Med Res. 2013;5:121-126.

19. Wang FM, Xu G, Zhang Y, et al. Red cell distribution width is associated with presence, stage, and grade in patients with renal cell carcinoma[J]. Dis Markers. 2014;2014:860419.

20. Qin Y, Wang P, Huang Z, et al. The value of red cell distribution width in patients with ovarian cancer[J]. Medicine (Baltimore). 2017;96:e6752.

21. Banzet $S$, Sanchez $H$, Chapot $R$, et al. Interleukin- 6 contributes to hepcidin mRNA increase in response to exercise[J]. Cytokine. 2012;58:158-161.

22. Wu Y, Antony S, Meitzler JL, et al. Molecular mechanisms underlying chronic inflammationassociated cancers[J]. Cancer Lett. 2014;345:164-173.

23. Li S, Meng W, Guan Z, et al. The hypoxia-related signaling pathways of vasculogenic mimicry in tumor treatment[J]. Biomed Pharmacother. 2016;80:127-135.

24. Cordella C, Luebbers HT, Rivelli V, et al. An evaluation of the preoperative hemoglobin level as a prognostic factor for oral squamous cell carcinoma[J]. Head Neck Oncol. 2011;3:35.

25. Tham T, Bardash Y, Teegala $S$, et al. The red cell distribution width as a prognostic indicator in upper aerodigestive tract (UADT) cancer: A systematic review and meta-analysis[J]. Am J Otolaryngol. 2018;39:453-458.

26. Hu L, Li M, Ding Y, et al. Prognostic value of RDW in cancers: a systematic review and metaanalysis[J]. Oncotarget. 2017;8:16027-16035. 


\section{Tables}

Table 1 Comparing demographic and experimental variables between patients with nasopharyngeal cancer and healthy controls.

\begin{tabular}{llll}
\hline Indicators & Nasopharyngeal cancer & Healthy controls & $P$ \\
\hline Number & 180 & 149 & \\
Age, years & $54.53 \pm 11.05$ & $53.65 \pm 10.44$ & 0.460 \\
Sex, Male:Female & $165: 15$ & $139: 10$ & 0.581 \\
Hemoglobin, g/L & $133.00 \pm 14.95$ & $150.21 \pm 6.34$ & $<0.001$ \\
Hematocrit & $0.41 \pm 0.04$ & $0.45 \pm 0.02$ & $<0.001$ \\
Plateletcrit & $0.23 \pm 0.06$ & $0.19 \pm 0.03$ & $<0.001$ \\
RDW, \% & $0.14 \pm 0.02$ & $0.13 \pm 0.01$ & 0.003 \\
Hb/RDW & $977.68 \pm 168.79$ & $1121.41 \pm 78.68$ & $<0.001$ \\
NLR & $2.99 \pm 2.11$ & $1.81 \pm 0.83$ & $<0.001$ \\
PLR & $159.54 \pm 82.71$ & $98.28 \pm 47.78$ & $<0.001$ \\
\hline
\end{tabular}

Data are expressed as mean \pm standard deviation (SD).

$P$-value was determined by the Turkey's test.

$\mathrm{Hb} / \mathrm{RDW}$, hemoglobin-to-red cell distribution width ratio (Hb/RDW); NLR, neutrophil-tolymphocyte ratio; PLR, platelet-to-lymphocyte ratio; RDW, Red blood cell distribution width

Table 2 Values of NLR, PLR, and Hb/RDW in 180 patients with nasopharyngeal cancer. 


\begin{tabular}{|c|c|c|c|c|c|c|c|}
\hline Indicators & $\mathrm{N}$ & $\mathrm{Hb} / \mathrm{RDW}$ & $P$ & NLR & $P$ & PLR & $P$ \\
\hline \multicolumn{8}{|l|}{ Stage } \\
\hline$\square+\square$ & 84 & $989.87 \pm 166.42$ & 0.152 & $2.67 \pm 1.73$ & 0.043 & $147.53 \pm 57.28$ & 0.424 \\
\hline$\square+\square$ & 96 & $967.02 \pm 171.00$ & & $3.27 \pm 2.37$ & & $170.05 \pm 98.92$ & \\
\hline \multicolumn{8}{|c|}{ Serosal invasion } \\
\hline $\mathrm{T}_{1}+\mathrm{T}_{2}$ & 56 & $1004.26 \pm 142.69$ & 0.182 & $2.57 \pm 1.68$ & 0.055 & $136.68 \pm 50.03$ & 0.031 \\
\hline $\mathrm{T}_{3}+\mathrm{T}_{4}$ & 124 & $965.68 \pm 178.58$ & & $3.18 \pm 2.26$ & & $169.87 \pm 92.15$ & \\
\hline \multicolumn{8}{|c|}{ Lymph node metastasis } \\
\hline Absence & 84 & $985.20 \pm 165.48$ & 0.301 & $2.66 \pm 1.74$ & 0.030 & $146.80 \pm 57.40$ & 0.313 \\
\hline Presence & 96 & $971.11 \pm 172.23$ & & $3.28 \pm 2.36$ & & $170.69 \pm 98.71$ & \\
\hline \multicolumn{8}{|c|}{ Distant metastasis } \\
\hline Absence & 171 & $983.11 \pm 166.97$ & 0.065 & $2.98 \pm 2.13$ & 0.570 & $158.43 \pm 79.29$ & 0.745 \\
\hline Presence & 9 & $874.65 \pm 180.30$ & & $3.25 \pm 1.81$ & & $180.71 \pm 137.72$ & \\
\hline
\end{tabular}

$P$-value was analyzed by the Kruskal-Wallis $\mathrm{H}$ test or the Wilcoxon rank-sum test. $\mathrm{Hb} / \mathrm{RDW}$, hemoglobin-to-red cell distribution width ratio (Hb/RDW); NLR, neutrophil-tolymphocyte ratio; PLR, platelet-to-lymphocyte ratio

Table 3 Correlation analysis of experimental indicators of 180 patients with nasopharyngeal cancer

\begin{tabular}{llll}
\hline Indicators & $\mathrm{Hb} / \mathrm{RDW}(P / \mathrm{r})$ & $\mathrm{NLR}(P / \mathrm{r})$ & $\mathrm{PLR}(P / \mathrm{r})$ \\
\hline $\mathrm{Hb} / \mathrm{RDW}$ & $*$ & $0.039 /-0.154$ & $0.039 /-0.154$ \\
$\mathrm{NLR}$ & $0.039 /-0.154$ & $*$ & $0.000 / 0.704$ \\
$\mathrm{PLR}$ & $0.039 /-0.154$ & $0.000 / 0.704$ & $*$ \\
Stage & $0.152 /-0.107$ & $0.043 / 0.151$ & $0.425 / 0.060$ \\
Serosal invasion & $0.184 /-0.100$ & $0.055 / 0.143$ & $0.031 / 0.161$ \\
Lymph node metastasis & $0.302 /-0.077$ & $0.030 / 0.162$ & $0.314 / 0.075$ \\
Distant metastasis & $0.064 /-0.138$ & $0.002 /-0.232$ & $0.002 /-0.227$ \\
\hline
\end{tabular}


$P$-value was calculated by the Pearson's or Spearman's correlation analysis. The r-value indicates the degree of correlation between the two related indicators.* Indicates that there is no correlation between the two indicators.

$\mathrm{Hb} / \mathrm{RDW}$, hemoglobin-to-red cell distribution width ratio (Hb/RDW); NLR, neutrophil-tolymphocyte ratio; PLR, platelet-to-lymphocyte ratio

Table 4 Diagnostic efficiency of NLR, PLR and Hb/RDW in patients with nasopharyngeal cancer and healthy controls.

\begin{tabular}{llllll}
\hline Indicators & Younder index & Sensitivity & Specificity & AUC & $95 \%$ CI \\
\hline NLR & 0.3975 & 60.56 & 79.19 & 0.734 & $0.683-0.781$ \\
PLR & 0.5016 & 58.89 & 89.28 & 0.809 & $0.763-0.850$ \\
$\mathrm{Hb} / \mathrm{RDW}$ & 0.519 & 66.67 & 85.23 & 0.781 & $0.732-0.824$ \\
$\mathrm{NLR}+\mathrm{Hb} / \mathrm{RDW}$ & 0.5704 & 67.78 & 89.26 & 0.824 & $0.779-0.864$ \\
$\mathrm{PLR}+\mathrm{Hb} / \mathrm{RDW}$ & 0.6338 & 72.78 & 90.60 & 0.851 & $0.808-0.888$ \\
\hline
\end{tabular}

AUC, area under the receiver operating characteristic (ROC) curve; 95\% CI, 95\% confidence interval; Hb/RDW, hemoglobin-to-red cell distribution width ratio; NLR, neutrophil-to-lymphocyte ratio; PLR, platelet-to-lymphocyte ratio

\section{Figures}

a

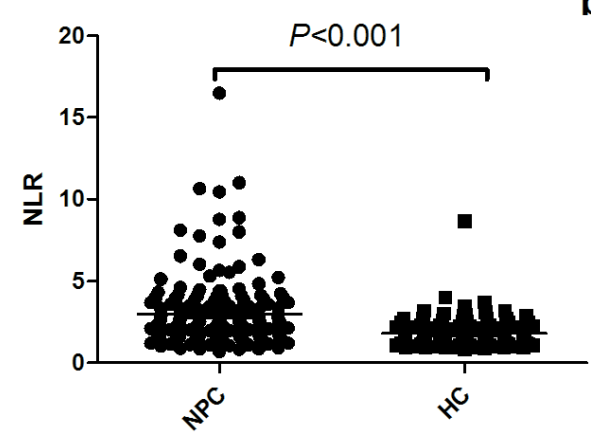

b

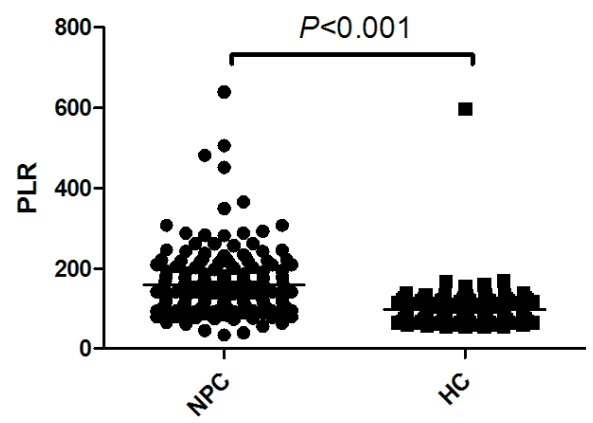

C

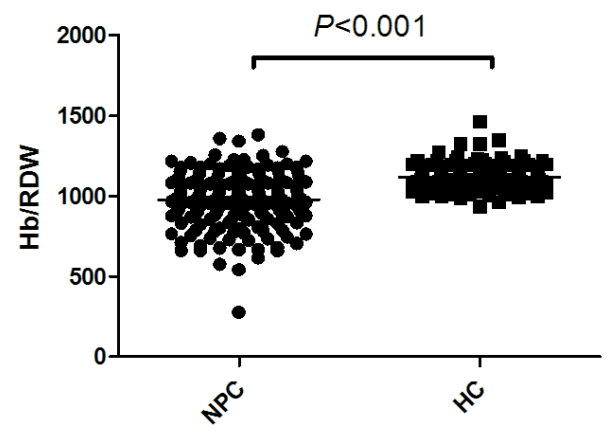

Figure 1 
Values of NLR, PLR, and Hb/RDWin the NPC group and HC group. a NLR in NPC group and HC group. b PLR in NPC group and HC group. $c \mathrm{Hb} / \mathrm{RDW}$ in NPC group and HC group. HC, healthy controls; Hb/RDW, hemoglobin-to-red cell distribution width ratio; NLR, neutrophil-to-lymphocyte ratio; NPC, nasopharyngeal cancer; PLR, platelet-to-lymphocyte ratio
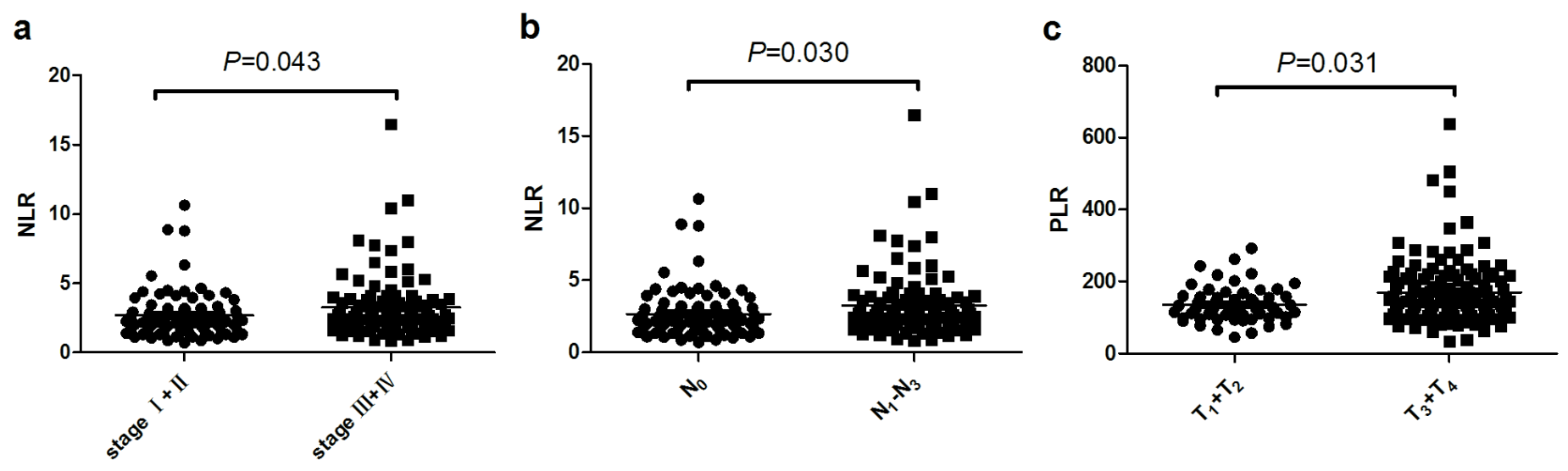

Figure 2

NLR and PLR in patients with nasopharyngeal cancer. a NLR in different stages. $b$ NLR in different lymph node metastases. c PLR in different serosal invasion. NLR, neutrophil-to-lymphocyte ratio; PLR, plateletto-lymphocyte ratio

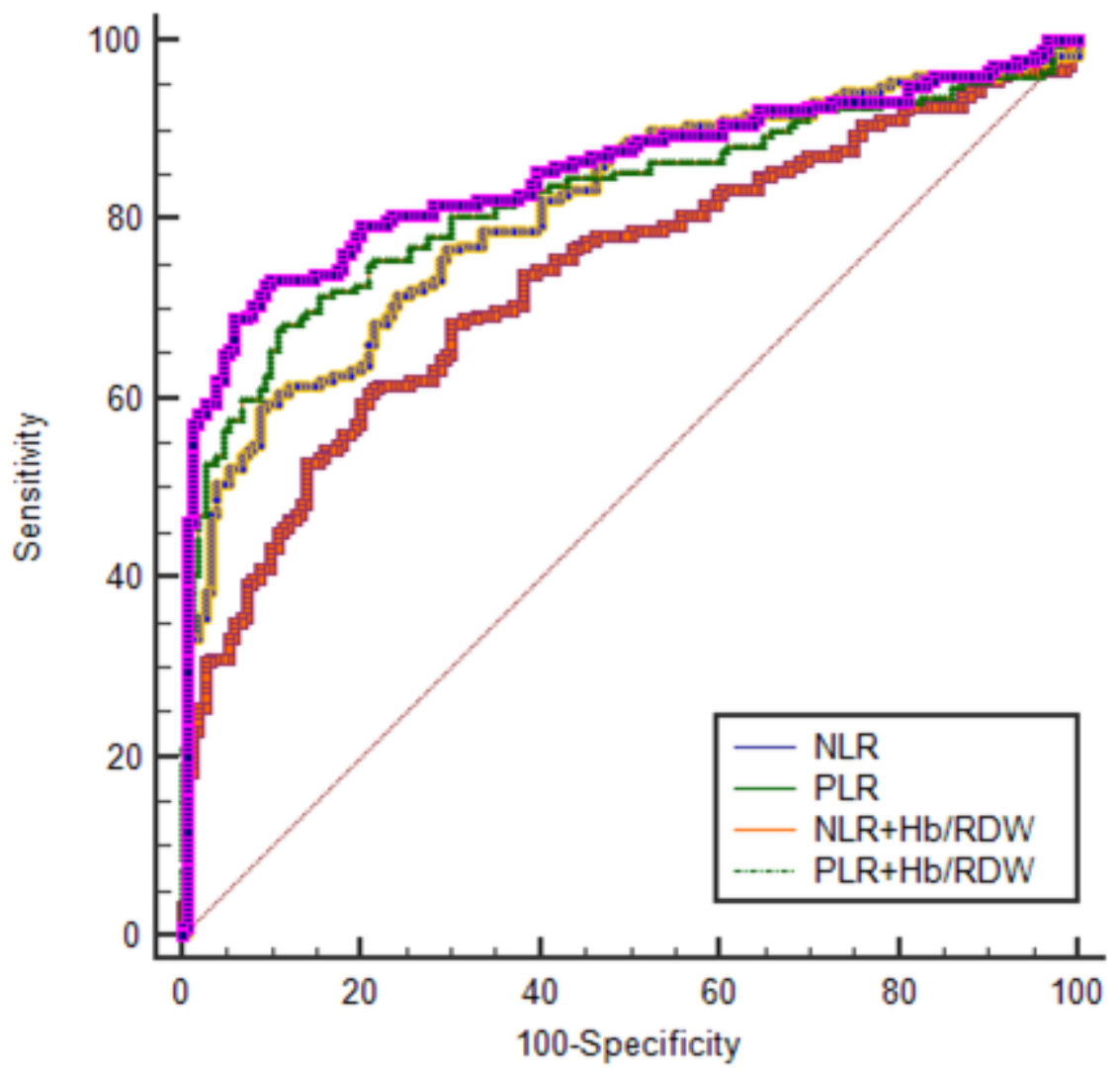




\section{Figure 3}

Combination of NLR, PLR and $\mathrm{Hb} / \mathrm{RDW}$ for the diagnosis of nasopharyngeal cancer. Hb/RDW, hemoglobin-to-red cell distribution width ratio; NLR, neutrophil-to-lymphocyte ratio; PLR, platelet-tolymphocyte ratio 